\title{
QUANTIFICATION OF GLOBAL OCULAR MOTILITY IMPAIRMENT IN GRAVES' ORBITOPATHY BY MEASURING EYE MUSCLE DUCTIONS
}

\author{
Irene Campi ${ }^{1,4} \S \mathrm{PhD}$, Nicola Currò ${ }^{2}$ MD§, Guia Vannucchi ${ }^{1} \mathrm{PhD}$, Danila Covelli ${ }^{3} \mathrm{MD}$, Simona \\ Simonetta ${ }^{2}$ BD, Laura Fugazzola ${ }^{1,4} \mathrm{MD}$, Davide Dazzi ${ }^{5} \mathrm{MD}$, Lorenzo Pignataro ${ }^{6} \mathrm{PhD}$, Claudio \\ Guastella ${ }^{6} \mathrm{MD}$, Elisa Lazzaroni ${ }^{3} \mathrm{MD}$, Giacinta Pirola ${ }^{2} \mathrm{BD}$ and Mario Salvi ${ }^{3} \mathrm{PhD}$. \\ ${ }^{1}$ Division of Endocrine and Metabolic Diseases \& Lab. of Endocrine and Metabolic \\ Research, IRCCS Istituto Auxologico Italiano, Piazzale Brescia n 20, 20145 Milan, Italy. \\ Telephone +3902 61911-2727 \\ ${ }^{2}$ Department of Surgery, Ophthalmology Unit, Fondazione IRCCS Ca' Granda, Ospedale \\ Maggiore Policlinico, via Francesco Sforza 35, 20122 Milan, Italy. +39 0255033912 \\ ${ }^{3}$ Graves' Orbitopathy Center, Endocrinology, Fondazione IRCCS Ca' Granda, Ospedale \\ Maggiore Policlinico, via Francesco Sforza 35, 20122 Milan, Italy. Telephone +39 02 \\ 55033332 \\ ${ }^{4}$ Department of Pathophysiology and Transplantation, University of Milan, via Francesco \\ Sforza 35, 20122 Milan, Italy. \\ ${ }^{5}$ Division of Internal Medicine, Ospedale Vaio, Via Don Enrico Tincati, 5, 43036 Fidenza, \\ Parma, Italy. Telephone +390524 515111 \\ ${ }^{6}$ ENT Unit, Fondazione IRCCS Ca' Granda, Ospedale Maggiore Policlinico, via Francesco \\ Sforza 35, 20122 Milan, Italy and Department of Clinical Sciences and Community \\ Health, University of Milan, via Francesco Sforza 35, 20122 Milan, Italy. Telephone +39 \\ 0255032563
}

Authors' e-mail addresses:

irene.campi@unimi.it; nicolacurro@virgilio.it; guiava@libero.it; simona.simonetta@unimi.it; laura.fugazzola@unimi.it; d.dazzi@icloud.com; lorenzo.pignataro@unimi.it; claudio.guastella@policlinico.mi.it; elisa.lazzaroni@hotmail.it; giacinta.pirola@policlinico.mi.it; mario@mariosalvinet.it.

$\S$ Irene Campi and Nicola Currò equally contributed to this work.

Running title: eye muscle ductions in Graves' orbitopathy

Key words: Graves' orbitopathy, ocular ductions, diplopia, rehabilitation, squint surgery, clinical trials. 
Corresponding Author : Irene Campi

IRCCS Istituto Auxologico Italiano, Ospedale S. Luca, Division of Endocrine and Metabolic Diseases, Piazzale Brescia n 20, 20145, Milan, Italy. Telephone: +39 02619112727 Fax +39

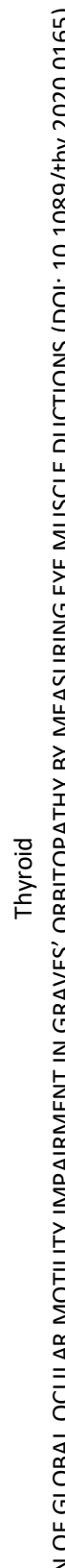

尊 02619112777

Disclosure Statement: No competing financial interests exist.

Funding: This work was supported by Fondazione IRCCS Cà Granda, Milan, Italy 
ABSTRACT

Background: The overall changes of ocular motility in Graves' Orbitopathy (GO), are not easily quantifiable with the methods currently available, especially in clinical studies. The aim of the present study was to calculate parameters that quantify the changes of ocular motility in GO, in relation to the Gorman score for diplopia.

Methods: We studied 100 GO patients (Group 1) and 100 controls (Group 2). We also included 30 patients treated with intravenous methylprednisolone (iv-MP) and assessed at baseline, after 12 and 24 weeks (Group 3) and 66 patients submitted to squint surgery, assessed at baseline and after 12 weeks (Group 4). Ocular ductions were measured in four gaze directions by a perimeter arc and were used to calculate a Total Motility Score (TMS), as the sum of ductions in each direction; a bi-ocular TMS (b-TMS) as the sum of the TMS of two eyes and an Asymmetry Ratio (AR) as the sum of the differences of the corresponding ductions between the two fellow eyes divided by the mean difference found in controls. Quality of life was accessed by a specific questionnaire (GO-Qol).

Results: TMS and b-TMS were lower, while AR was higher in Group 1 compared to controls $(\mathrm{P}<0.001)$. In Group 1, TMS and b-TMS were inversely correlated with the Gorman score $(P<0.001)$ and $A R$ was higher in patients with constant diplopia compared to the others $(P<0.001)$. In Group 3, TMS and b-TMS increased after treatment in responders to iv-MP $(P<0.001)$. In Group 4, TMS and b-TMS improved in all patients after surgery $(P<0.01)$, while $A R$ and GO-Qol improved only in those without residual constant diplopia $(P<0.001)$. Conclusion: We describe a quantitative method to assess the eye motility dysfunction in any stage of GO to be used as an outcome measure in clinical studies. 


\section{INTRODUCTION}

Graves' Orbitopathy (GO) has a very heterogeneous presentation and its impact on the patients' quality of life is universally recognized, especially when extraocular motility is affected. $(1,2)$ The assessment of extra-ocular motility is of paramount importance when grading the severity of $\mathrm{GO}$ and when assessing the outcomes of treatment but, unfortunately, the overall modifications of eye muscle function are not easily quantifiable with the methods currently available, especially in clinical studies.

The precise measurement of ocular ductions (the movements of one eye while the contralateral is occluded) is recommended when assessing motility, but this approach has some limitations. For example, the improvement of ductions in one direction of gaze after immunosuppression, if accompanied by deterioration in other gazes, may result in worse subjective diplopia and a decrease of the patients' quality of life, even if therapy has successfully inactivated GO.

When evaluating the effectiveness of medical treatment or surgical procedures, the primary endpoint should ideally be measurable as a continuous parameter in order to allow accurate and reliable statistical analysis. In common clinical practice, such measures may however be difficult to obtain, and the outcomes are often reported as dichotomous or discrete parameters, such as active/inactive disease, presence/absence of diplopia. As an example, the Gorman score for diplopia has been the most commonly used parameter in randomized clinical trials studying GO $(3,4)$ This score grades diplopia into 4 classes according to its severity (absent, intermittent, gaze evoked or constant) but its categorical nature implies some statistical limitations.

In the present study we measured parameters derived from ocular ductions in a series of patients with different grades of subjective diplopia. We have subsequently applied these parameters as outcome measures in randomized clinical trials with steroid therapy for active GO and after surgical correction of strabismus. These numerical continuous parameters may offer a large variety of statistical analysis options especially valid in small cohort studies, typical of GO, a rare disease. To be reliable, these parameters should meet the following criteria: a) to be significantly different in GO patients with diplopia and controls; b) to correlate with diplopia graded with the Gorman score; c) to correlate with the patient's quality of life. 


\section{METHODS}

\section{Patients}

We studied consecutive GO patients and controls seen at our joint Thyroid Eye Clinic from January 2015 to April 2017 and controls. The research complied with the Declaration of Helsinki and was approved by our Institutional Review Board (11/22/2018, n. 2260). Patients were divided into four groups according to their clinical presentation:

Group 1: 100 GO patients, 74 women and 26 men (aged 15-82 years, mean $53 \pm 13.5$ yr); included in the group were the first 25 consecutive patients for each class of the Gorman score. Patients had either inactive $(n=60)$ or active $(n=40)$ GO and of the latter, 36 had moderate-severe disease with a mean Clinical Activity Score (CAS) of $4.1 \pm 1$ (7).

Group 2: 100 healthy volunteers, 74 women and 26 men (aged 20-79 years, mean 49 \pm 16.1 yr).

Group 3: 30 patients, eight men and 22 women (aged 27-78 years, mean $53 \pm 12 \mathrm{yr}$ ) euthyroid on methimazole treatment, with active moderate-severe GO (mean CAS 4.6 \pm 1.2 ), undergoing treatment with intravenous methylprednisolone (iv-MP) and receiving a cumulative dose of $7.5 \mathrm{~g}$. They were studied at baseline, at the end of treatment (12 weeks) and at 24 weeks of follow-up. Patients with a CAS $<3$ at 24 weeks, were considered responders to steroids.

Group 4: 66 euthyroid patients, 24 men and 42 women (aged 24-85 years, mean $58.4 \pm 12$ yr) with inactive GO, studied at baseline and 12 weeks after squint surgery for constant diplopia. Patients without diplopia in straight and downgaze at 12 weeks, were considered responders to surgery.

None of the patients were previously treated with orbital radiotherapy.

\section{Calculation of TMS, bi-ocular TMS and AR}

We calculated the Total Motility Score (TMS), the bi-ocular TMS (b-TMS) and the Asymmetry Ratio (AR) based on the values of ocular ductions, measured (in degrees) in the four main orthogonal gaze directions (up gaze, abduction, downgaze, adduction) with a Förster perimeter arc $(5,6)$ (Supplemental Figure 1A). The TMS was calculated for each eye as the sum of the values of ductions, as previously reported (7) The b-TMS was calculated as the sum of the TMS of the right and of the left eye of each patient. 
The AR, which is an estimate of the misalignment of the two fellow eyes and was calculated as the sum of the differences of the corresponding ductions of the two fellow eyes in each gaze direction divided by the mean difference of the ductions found in controls $\left(=5.2^{\circ}\right)$. A physiological asymmetry of motility between the two eyes was found in normal individuals (Group 2) as the mean difference of the corresponding ductions in these subjects was 5.2 degrees (Supplemental Figure $1 \mathrm{~A}$ ). Consequently, the AR is expressed as a ratio of the mean physiological asymmetry and is expected to be 0 in the few individuals with a perfect symmetrical motility between the right and the left eye and around 1 in the majority of controls (Group 2) (Supplemental Figure 1B). Conversely, GO patients with diplopia are expected to have an $A R>1$ (Supplemental Figure $1 C$ and D).

The presence of subjective diplopia was recorded according to the Gorman score for diplopia (0=no diplopia; 1=intermittent diplopia; 2=inconstant or gaze evoked diplopia; $3=$ constant diplopia). The Clinical Activity Score (CAS) (8) was assessed in all patients of Group 3, throughout follow up. We first studied the relationship of TMS, b-TMS and AR with the Gorman score and with the patients' quality of life, in order to validate the measures in different grades of disease, and subsequently their changes in response to both medical and surgical treatments.

\section{Quality of life}

The GO quality of life (GO-Qol) questionnaire (8) was administered to all patients of Group 1, 2 and 3 and was returned by 82/100 (82\%), 26/30 (86\%) and 27/66 (41\%) of patients, respectively. Both the visual functioning and appearance scores, as previously reported by Terwee et al. (9), were calculated and correlated with b-TMS and AR.

\section{Statistical considerations}

According to the results of previous studies by Haggerty (5), Gerling (6) and Mourits (10), the mean reduction of TMS between GO patients with diplopia and controls can be estimated to be between 19 and 27\% (Supplemental Table 1).

Assuming that patients with GO without muscle involvement may have TMS values comparable to that of controls, we tested the hypothesis that GO patients with constant diplopia may have a $20 \%$ reduction of TMS compared with those without diplopia. To detect this difference with $80 \%$ power and a significance level of 0.05 , the sample size should be of at least 19 subjects for each group. 
Thus, we included in the study 100 patients, divided into 4 groups of 25 patients for each class of diplopia and compared them to a group of 100 healthy controls.

All values are expressed as mean \pm SD. The limits of normal values of TMS and bi-ocular TMS were calculated as the mean \pm 2 SD of the values of normal controls. Analysis by one way and repeated-measures ANOVA (with Bonferroni correction), Friedman test or Chi square test, Wilcoxon signed rank or Mann-Whitney test and the Spearman correlation tests was applied, as appropriate. ROC analysis was used to determine the cut-off values for the AR, chosen to maximize specificity.

\section{RESULTS}

\section{Assessment of TMS, b-TMS and AR.}

In GO patients (Group 1) and controls (Group 2) the mean values of ductions and TMS measured in the right eye were not significantly different from those measured in left eye, in any of the gazes analyzed. As expected, GO patients had mean ductions values in all but downgaze significantly reduced, when compared to controls $(P<0.001)$ (Table 1$)$. Mean TMS and b-TMS values in GO patients were $14 \%$ lower than those of normal subjects $(P<0.001)$. The range of normal values (mean $\pm 2 S D)$ for TMS and $b-T M S$ was calculated as 192-232 and 388-456 degrees, respectively. By ROC curve analysis, we determined a cutoff value of $198^{\circ}$ and $396^{\circ}$ for the TMS an b-TMS, respectively, which best differentiated patients with GO from normal subjects (65\% sensitivity and $90 \%$ specificity, for both values, data not shown).

In contrast, mean AR in GO patients was three times as high as in controls. (Table 1; $P<0.001)$. This parameter had large variability due to the different degree of diplopia in Group 1, ranging from absent to constant diplopia. By ROC curve analysis, the optimal cutoff value was 2.64 for AR in patients with diplopia compared to normal subjects. This value had $100 \%$ specificity.

\section{Relationship between b-TMS and AR, diplopia and GO-QoL.}

In order to determine the relationship between b-TMS, AR and diplopia, we calculated these parameters in patients with GO with different classes of diplopia according to the Gorman score. The values of ductions, TMS and b-TMS of GO patients without diplopia were not significantly different from those of control (Table 2). The values of ductions in all 
but downgaze, were found to be significantly lower with higher Gorman score grading (Table 2).

Mean b-TMS was 34 degrees (7\%), 66 degrees (15\%), and 116 degrees (27\%) lower in patients with intermittent, inconstant and constant diplopia, respectively, when compared with the $b$-TMS of patients without diplopia (Figure $1 \mathrm{~A}, \mathrm{P}<0.05$ ).

The AR was significantly higher in patients with constant diplopia (Gorman score $=3$ ), when compared to controls (Table 2) or to patients with lower grades of diplopia (Figure 1B; $\mathrm{P}<0.001)$.

The mean $b-T M S$ was negatively (Spearman $R=-0.86, P<0.001$; data not shown) and, as expected, the AR positively correlated with the Gorman score (Spearman $R=0.65, P<0.001$; data not shown).

In GO patients, a high diplopia score was significantly associated to a lower score of visual functioning on the GO-Qol (Figure 2A), and the two parameters were negatively correlated (Spearman $\mathrm{R}=-0.67 ; \mathrm{P}<0.001$, not shown). As shown in Figure 2, the b-TMS was positively correlated (2B) and the AR negatively correlated (2C) with the visual function score of the GO-Qol.

\section{Changes of TMS, b-TMS and AR in response to immunosuppressive therapy.}

After having found a relationship between the motility parameters (TMS, b-TMS and AR) and the degrees of diplopia, we studied the usefulness of these parameters in assessing the outcome of steroid immunosuppressive therapy on extra-ocular motility and that of strabismus surgery.

Twenty-four of 30 patients (80\%) with active GO responded to iv-MP therapy, with a significant reduction of CAS compared to baseline $(p<0.001$ Table 3$)$. Despite disease inactivation, at 24 weeks their diplopia did not improve when assessed with the Gorman score or with the AR (Table 3). In contrast, the TMS and the b-TMS calculated at 24 weeks increased significantly $(P<0.001)$ compared to baseline values. We also observed a significant improvement in the GO-Qol. In the six patients unresponsive to iv-MP (20\%), the TMS and the b-TMS did not change, nor did their GO-Qol (Table 3). At baseline, the AR was higher in the patients not responding to steroids and, although it improved at 24 weeks after therapy, its mean value was still more than two times the upper limit of normal (Table 
3). Interestingly, 14 of 30 patients (46.6\%), in whom b-TMS normalized after iv-MP treatment, did not require additional medical or surgical treatment (data not shown).

\section{Eye motility changes in response to squint surgery.}

Sixty-six GO patients who underwent rehabilitative surgery for constant diplopia (Group 4) were assessed at baseline and 12 weeks after surgery. Thirty-six patients (54.5\%) responded to surgery and had no diplopia in straight and downgaze at 12 weeks. In these patients, the Gorman score, TMS, b-TMS, AR and GO-Qol significantly improved after surgery (Table 4$)$. The 30 patients (45.5\%) not responding to squint surgery had constant diplopia after surgery and despite the improvement of the TMS and the b-TMS $(P<0.001)$, their $A R$ and GO-Qol score did not improve (Table 4). Ten non-responders were satisfactorily corrected with low power prismatic lenses, while the remaining 20 were scheduled for additional surgery.

\section{DISCUSSION}

In this study, we calculated three parameters of extra-ocular motility (TMS, b-TMS and AR), based on the measurement of ocular ductions. Ductions recorded in patients and controls showed to be consistent with those reported by Gerling et al (6) and by Haggerty et al (5), suggesting that these parameters can be accurate and reproducible measurements when applied to different cohorts of patients affected with different grades of severity of GO.

The TMS, calculated as the sum of the values of the ocular ductions, is an index of the motility impairment of each single eye of a given patient. Accordingly, the bi-ocular TMS (the sum of the values of the TMS of the two fellow eyes) is an index of the patients' global motility involvement (both eyes). We have also tried to study and quantify the degree of diplopia, being aware of its impact on the patients' quality of life, by calculating the $A R$, an objective index of the asymmetry between the extra-ocular motility of the two eyes.

The study of ocular ductions is usually performed by observation and grading during the routine orthoptic examination. (11) More precise measurements can be obtained by several objective techniques, such as the corneal reflex method, (12) the limbus test of Kestenbaum, (13) the use of perimetry (Goldmann perimeter or Förster perimeter arc) $(6,12)$, the Harms tangent screen (14), the Maddox tangent scale (15) or the cervical range of motion. $(16,17)$ These instruments can be ideally used to calculate TMS, b-TMS and AR, 
as long as the same tool is used for patients' follow up. To date, changes of ductions over time and in response to treatment have been determined by measuring the ductions recorded in each different direction of gaze, eye by eye. $(6,12,18,19)$. Unfortunately this approach may lead to a standstill when judging the effects of medical treatments on ocular motility, particularly when the improvement in one or more directions is accompanied by simultaneous deterioration in other directions of gaze. In this prospect, the calculation of TMS and b-TMS may help provide a global measure of ocular motility. Furthermore, when treating GO patients with deterioration of the ocular motility, the measurement of changes of TMS and b-TMS may help assess the effects of therapy.

The assessment of diplopia can be based on either subjective or objective methods (20, 21). Subjective methods are still the most commonly used in both clinical practice and clinical trials. These methods classify diplopia as reported by the patient, according to discrete, ordinal scores. Among them the Gorman score (6) and the VISA diplopia score (8) have been the most frequently used.

Among methods that objectively assess diplopia, the field of diplopia is currently considered the gold standard (21). It is more frequently plotted using a Goldman perimeter, (22) although other technical devices have been proposed $(23,24)$. Within the binocular field of vision, the field of diplopia delimits the area of diplopia from that of single vision. Unfortunately, the results of this examination are commonly assessed as improved or worsened, while the area of diplopia is rarely measured, due to the complexity of the calculation. Other scoring systems for the quantification of the field of diplopia have been proposed (24), but they are particularly time consuming.

As shown in the present work, both b-TMS and AR are significantly related to subjective diplopia. The fact that, in patients with GO, diplopia is usually related to progressive restriction of ocular motility explains why the b-TMS decreases and the AR increases with the increase of the diplopia score grading. Our data show that the likelihood to have diplopia increases with the worsening of the overall ocular motility, although the b-TMS does not allow distinguishing between symmetrical and asymmetrical eye muscle involvement. Impairment of the eye muscles in a cohort of patients with GO is generally asymmetrical, but patients with low b-TMS and no diplopia may have symmetrical limitation of ocular motility. The increase of AR was shown to be significant only in 
patients with the highest grade of the Gorman score (constant diplopia). These findings are strengthened by the observation that in patients undergoing strabismus surgery, the AR improved significantly only in those who successfully responded to surgical correction of diplopia (no diplopia in primary position and downgaze) but did not in patients with residual constant diplopia after surgery, even when ductions improved. By ROC curve analysis we were able to determine the cut-off value for AR at 2.64 with $100 \%$ specificity when comparing GO patients of Group 1 to controls. The low sensitivity of the test (44\%) may be explained by the presence of GO patients without diplopia, as the AR of these patients does not differ from that of controls (Table 2). By excluding GO patients with a Gorman score 0 (no diplopia), the test increases sensitivity to $54 \%$ with a better AUC (0.77) (data not shown).

Since diplopia is among the major causes of decreased quality of life in patients with GO, we also studied the relationship between b-TMS, AR and the patients' quality of life as assessed by the GO-Qol (9). As expected, we have found a decrease of the quality of life in patients with a higher degree of diplopia as measured by the Gorman score grading. Specifically, the visual functioning score of the GO-QOL (which measures the impact of double vision on daily life activities) but not the appearance score (which mainly relates to cosmetic changes) decreased with a higher score of diplopia in patients in Group 1. Such inverse correlation was observed also with the AR, while the b-TMS was positively correlated with the visual function score of the GO-Qol. This suggests that the patients' quality of life is not only associated to subjective diplopia, as expected, but can also be related to objective quantitative parameters of extra-ocular motility.

A limitation of the study is that a comparison between the AR and the field of diplopia that was not performed in the training group of patients, who were studied retrospectively. In addition, although a perimeter arc or a Goldman bowl are very simple and widespread instruments, they are currently not easily available, as they have been replaced by the more modern automated perimeters used in the assessment of the visual field. However, any instrument capable of measuring ductions can adequately calculate TMS, b-TMS and $A R$ as long as ductions are measured with the same device over time.

In conclusion, the measurement of ductions provides a global assessment of eye muscles function in GO. The TMS, b-TMS and AR are quantitative parameters that can be used as 
outcomes of clinical trials, allowing a more gradual and objective measure of the changes of the ocular motility, as compared with the categorical variables currently in use.

\section{ACKNOWLEDGMENTS}

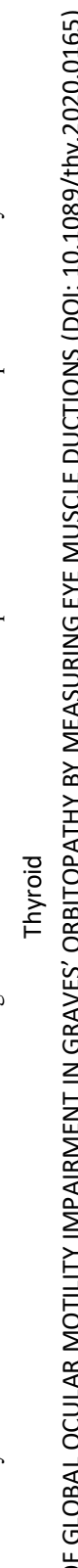

We thank Sabina di Benedetto, Alessandra Bottari de Castello, Barbara Calonghi, Paola Acanti and Valeria Minorini for the assistance in the orthoptic assessment of the patients and controls included in the present study. 


\section{REFERENCES}

1 Kahaly GJ, Petrak F, Hardt J, Pitz S, Egle UT 2005 Psychosocial morbidity of Graves' orbitopathy. Clin Endocrinol 63:395-402.

2 Hatt SR, Leske DA, Kirgis PA, Bradley EA, Holmes JM 2007 The effects of strabismus on quality of life in adults. Am J Ophthalmol 144:643-647.

3 Kahaly GJ, Riedl M, König J, Pitz S, Ponto K, Diana T, Kampmann E, Kolbe E, Eckstein A, Moeller LC, Führer D, Salvi M, Curro N, Campi I, Covelli D, Leo M, Marinò M, Menconi F, Marcocci C, Bartalena L, Perros P, Wiersinga WM; European Group on Graves' Orbitopathy (EUGOGO) 2018 Mycophenolate plus methylprednisolone versus methylprednisolone alone in active, moderate-to-severe Graves' orbitopathy (MINGO): a randomised, observer-masked, multicentre trial. Lancet Diabetes Endocrinol 6:287-298.

4 Bartalena L, Krassas GE, Wiersinga W, Marcocci C, Salvi M, Daumerie C, Bournaud C, Stahl M, Sassi L, Veronesi G, Azzolini C, Boboridis KG, Mourits MP, Soeters MR, Baldeschi L, Nardi M, Currò N, Boschi A, Bernard M, von Arx G; European Group on Graves' Orbitopathy 2012 Efficacy and safety of three different cumulative doses of intravenous methylprednisolone for moderate to severe and active Graves' orbitopathy. J Clin Endocrinol Metab 97:4454-4463.

5 Haggerty H, Richardson S, Mitchell K, Dickinson AJ 2005 A modified method for measuring uniocular fields of fixation: reliability in healthy subjects and in patients with Graves orbitopathy. Arch Ophthalmol 123:356-362.

6 Gerling J, Lieb B, Kommerell G 1998 Duction ranges in normal probands and patients with Graves' ophthalmopathy, determined using the Goldmann perimeter. Int Ophthalmol 21:213-221.

7 Salvi M, Vannucchi G, Currò N, Covelli D, Dazzi D, Simonetta S, Guastella C, Pignataro L, Avignone S, Beck-Peccoz P 2015 Efficacy of B-cell targeted therapy with rituximab in patients with active moderate to severe Graves' orbitopathy: a randomized controlled study. J Clin Endocrinol Metab 100:422-431.

8 Mourits MP, Koornneef L, Wiersinga WM, Prummel MF, Berghout A, van der Gaag R 1989 Clinical criteria for the assessment of disease activity in Graves' ophthalmopathy: a novel approach. Br J Ophthalmol 73:639-644. 
9 Terwee CB, Gerding MN, Dekker FW, Prummel MF, Wiersinga WM 1998 Development of a disease specific quality of life questionnaire for patients with Graves' ophthalmopathy: the GO-QOL. Br J Ophthalmol 82:773-779.

10 Mourits MP, Prummel MF, Wiersinga WM, Koornneef L 1994 Measuring eye movements in Graves ophthalmopathy. Ophthalmology 101:1341-1346.

11 Jellema HM, Baader A, Pitz S, Wiersinga WM, Mourits MP 2011 Comparison of cyclodeviation and duction measurement in Graves' orbitopathy patients using different devices. Strabismus 19:43-51.

12 Dolman PJ, Cahill K, Czyz CN, Douglas RS, Elner VM, Feldon S, Kazim M, Lucarelli M, Sivak-Collcott J, Stacey AW, Strianese D, Uddin J 2012 Reliability of estimating ductions in thyroid eye disease: an International Thyroid Eye Disease Society multicenter study. Ophthalmology 119:382-389.

13 Kupersmith MJ1, Fazzone HE 2004 Comparing ocular muscle limitation tests for clinical trial use. Arch Ophthalmol 122:347-348.

14 Hanif S, Rowe F, O'Connor A 2009 A comparative analysis of monocular excursion measures. Strabismus 17:29-32

15 Kushner BJ 2000 The usefulness of the cervical range of motion device in the ocular motility examination. Arch Ophthalmol 118:946-50.

16 Dolman PJ, Rootman J 2006 VISA Classification for Graves orbitopathy. Ophthalmic Plast Reconstr Surg 22:319-324.

17 Jellema HM, Saeed P, Mombaerts I, Dolman PJ, Garrity J, Kazim M, Dhrami-Gavazi E, Lyons C, Nieuwkerk P, Mourits MP 2017 Objective and subjective outcomes of strabismus surgery in Graves' orbitopathy: a prospective multicentre study. Acta Ophthalmol 95:386-391.

18 Kestenbaum A 1961 Clinical Methods of Neuro-ophthalmological Examinations. 2nd ed.: Grune \& Stratton; New York, NY

19 Gutter M, van Petegem-Hellemans J, van Wijnen-Segeren I, Jellema HM 2010 Orthoptics: Handbook of Practical Skills. Ridderkerk NL Luiten publishing.

20 Mourits MP, Koorneef L, van Mourik-Noordenbos AM, van der Meulen-Schot HM, Prummel MF, Wiersinga WM, Berghout A 1990 Extraocular muscle surgery for 
Graves' ophthalmopathy: does prior treatment influence surgical outcome? $\mathrm{Br} \mathrm{J}$ Ophthalmol 74:481-483.

21 Fitzsimons R, White J 1990 Functional scoring of the field of binocular single vision. Ophthalmology 97:33-35.

22 Bartalena L, Baldeschi L, Dickinson AJ, Eckstein A, Kendall-Taylor P, Marcocci C, Mourits MP, Perros P, Boboridis K, Boschi A, Currò N, Daumerie C, Kahaly GJ, Krassas G, Lane CM, Lazarus JH, Marinò M, Nardi M, Neoh C, Orgiazzi J, Pearce S, Pinchera A, Pitz S, Salvi M, Sivelli P, Stahl M, von Arx G, Wiersinga WM 2008 Consensus statement of the European group on Graves' orbitopathy (EUGOGO) on management of Graves' orbitopathy. Thyroid 18:333-346.

23 Sullivan TJ, Kraft SP, Burack C, O'Reilly C 1992 A functional scoring method for the field of binocular single vision. Ophthalmology 99:575-581.

24 Woodruff G, O'Reilly C, Kraft SP 1987 Functional scoring of the field of binocular single vision in patients with diplopia. Ophthalmology 94:1554-1561. 
FIGURE LEGENDS
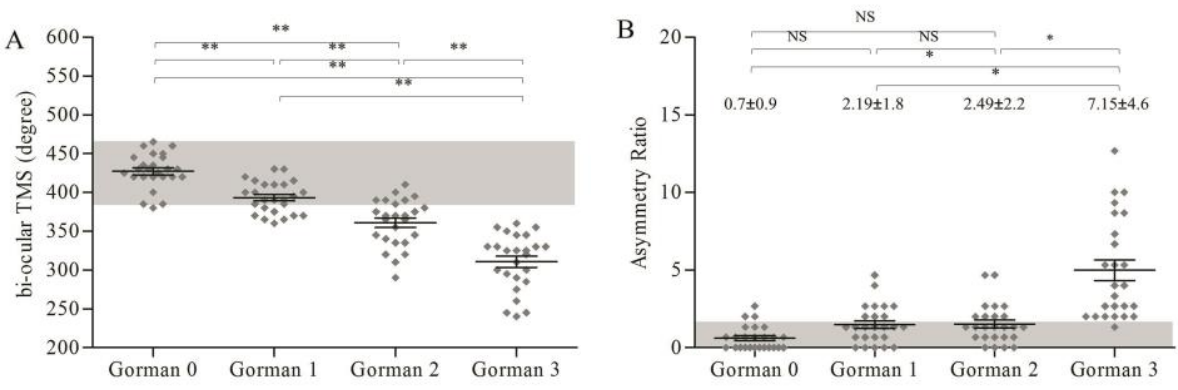

Figure 1: Relationship between the Gorman score and motility parameters quality of life in 100 patients with Graves' Orbitopathy (Group 1). A: Relationship between the Gorman score and b-TMS (biocular Total Motility Score) B: Relationship between the Gorman score and AR (Asymmetry Ratio)

${ }^{*}=p<0.05 ;{ }^{* *}=P<0.001 ; \mathrm{NS}=$ not significant. 

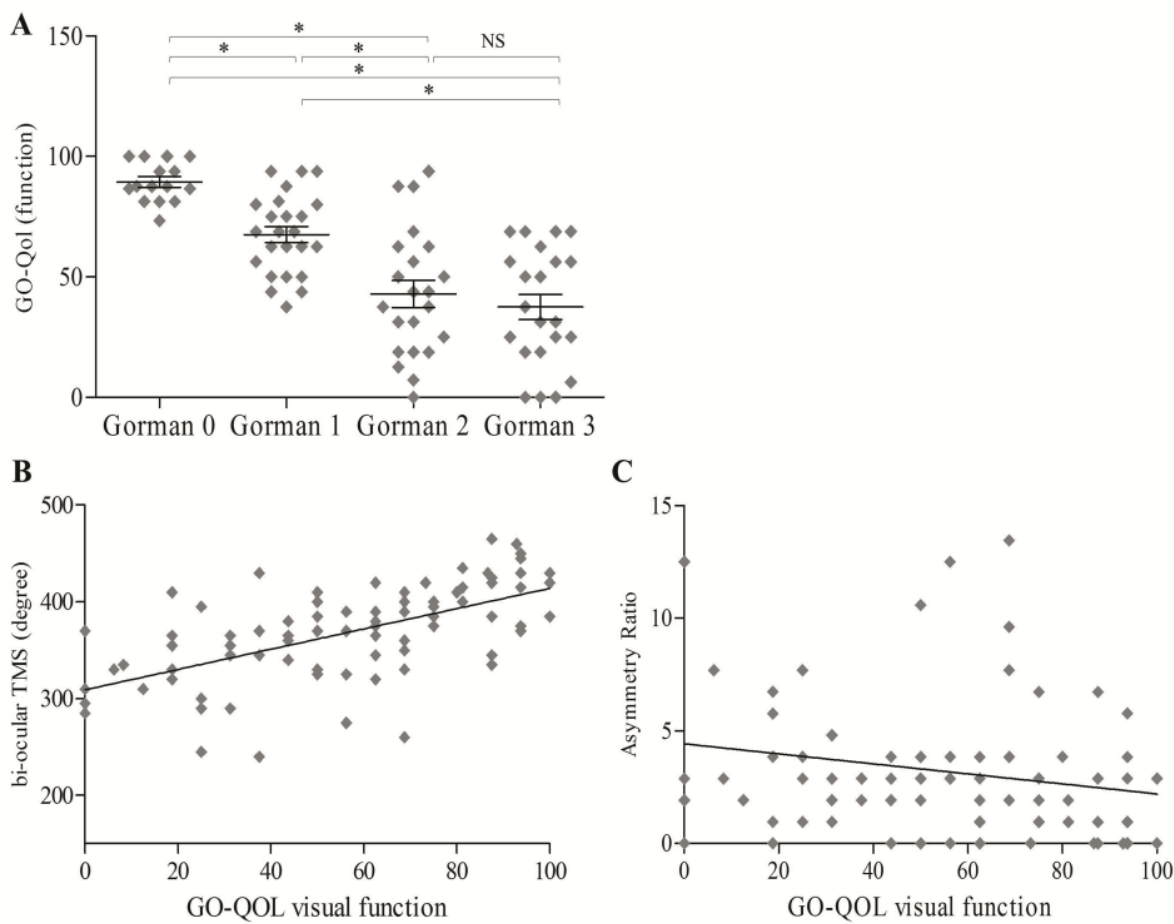

Figure 2: Correlation between motility parameters and the visual functioning score of GOQoL in 100 patients with Graves' Orbitopathy (Group 1). A: Relationship between the Gorman score and the visual function score of GO-QoL (Graves' Orbitopathy Quality of Life questionnaire). One way ANOVA $*=p<0.05$. B: Correlation between the visual function score of the GO-QoL and the Biocular Total Motility score (b-TMS); Spearman R=0.64, $\mathrm{P}<0.001$. C: Correlation the visual function score of the between GO-QoL and Asimmetry Ratio (AR); Spearman $R=-0.3, p<0.05$. 
Table 1: Clinical characteristics and values of ductions (in degrees) measured in 100 patients with GO (Group 1) and 100 normal controls (Group 2).

\begin{tabular}{lccc}
\hline & Group 1 & Group 2 & $\boldsymbol{P}$ \\
\hline Age (years) & $53 \pm 13.5$ & $49 \pm 16.1$ & NS* \\
F/M (n) & $74 / 26$ & $74 / 26$ & NS*
\end{tabular}

Right eye Left eye Right eye Left eye

Upgaze

Downgaze

Abduction

Adduction

TMS

b-TMS

Differences in

ductions between

eyes

AR

$3.17 \pm 3.63^{\circ}$

$15.9 \pm 18.7^{\circ}$

$5.201 \pm 2.8^{\circ}$

$<0.001^{* *}$

$\mathrm{F}=$ female; $\mathrm{M}=$ male; TMS = total motility score; $\mathrm{b}-\mathrm{TMS}=$ bi-ocular TMS; $\mathrm{AR}=$ asymmetry ratio, $\mathrm{SD}=$ standard deviation

* Chi-square test ** Mann Whitney test

Values are presented as mean \pm standard deviation, unless otherwise noted. 
Table 2. Relationship between ductions values (in degrees) and diplopia in $100 \mathrm{GO}$ patients selected according with their Gorman score (Group 1) and normal controls (Group 2).

\begin{tabular}{|c|c|c|c|c|c|c|c|}
\hline & & \multirow[t]{3}{*}{$\begin{array}{l}\text { Controls* } \\
(n=100)\end{array}$} & \multicolumn{4}{|c|}{$\begin{array}{l}\text { GO patients } \\
(n=100)\end{array}$} & \multirow[t]{3}{*}{$P$} \\
\hline & & & \multirow[b]{2}{*}{$\begin{array}{c}0^{*} \\
(n=25)\end{array}$} & \multicolumn{2}{|c|}{ Gorman score } & \multirow[b]{2}{*}{$\begin{array}{c}3 \\
(n=25)\end{array}$} & \\
\hline & & & & $\begin{array}{c}1 \\
(n=25)\end{array}$ & $\begin{array}{c}2 \\
(n=25)\end{array}$ & & \\
\hline \multirow{2}{*}{ Upgaze } & $\mathrm{RE}$ & $38 \pm 4$ & $36 \pm 5$ & $31 \pm 7$ & $21 \pm 10$ & $11 \pm 12$ & $<0.005$ \\
\hline & LE & $38 \pm 4$ & $37 \pm 5$ & $31 \pm 8$ & $21+9$ & $14 \pm 16$ & $<0.001$ \\
\hline \multirow{2}{*}{ Downgaze } & RE & $63 \pm 3$ & $65 \pm 2$ & $63 \pm 5$ & $63 \pm 3$ & $61 \pm 5$ & NS \\
\hline & LE & $63 \pm 3$ & $65 \pm 1$ & $63 \pm 4$ & $63 \pm 3$ & $60 \pm 9$ & NS \\
\hline \multirow{2}{*}{ Abduction } & RE & $56 \pm 3$ & $58 \pm 5$ & $53 \pm 6$ & $51 \pm 6$ & $40 \pm 12$ & $<0.001^{\#+}$ \\
\hline & LE & $56 \pm 2$ & $58 \pm 6$ & $54 \pm 4$ & $48 \pm 7$ & $40 \pm 10$ & $<0.001^{+}$ \\
\hline \multirow{2}{*}{ Adduction } & RE & $55 \pm 2$ & $54 \pm 3$ & $49 \pm 6$ & $47 \pm 6$ & $41 \pm 8$ & $<0.001^{\#}$ \\
\hline & LE & $54 \pm 2$ & $54 \pm 6$ & $48 \pm 4$ & $47 \pm 6$ & $43 \pm 8$ & $<0.001^{\#}$ \\
\hline \multirow{2}{*}{ TMS } & RE & $212 \pm 10$ & $213 \pm 11$ & $196 \pm 13$ & $180 \pm 16$ & $154 \pm 26$ & $<0.001$ \\
\hline & LE & $210 \pm 9$ & $214 \pm 12$ & $197 \pm 11$ & $180 \pm 16$ & $157 \pm 27$ & $<0.001$ \\
\hline b- TMS & & $422 \pm 17$ & $427 \pm 22$ & $393 \pm 21$ & $361 \pm 31$ & $311 \pm 36$ & $<0.001$ \\
\hline$A R$ & & $1.0 \pm 0.5$ & $0.73 \pm 0.9$ & $2.19 \pm 1.8$ & $2.65 \pm 2.3$ & $7.15 \pm 4.6$ & $<0.001^{\S}$ \\
\hline
\end{tabular}

The number of patients for each group is indicated in brackets.

RE: right eye; LE: left eye; TMS: total motility score, b-TMS=bi-ocular TMS; AR: asymmetry ratio; $\mathrm{SD}=$ standard deviation. $\mathrm{P}=\mathrm{NS}$

Test one-way ANOVA, post test Bonferroni's Multiple Comparison Test.All values are expressed as mean \pm SD.

* no significant differences found between controls and patients without diplopia (Gorman $0)$. \# no significant differences found between Gorman 1 and 2 . + no significant differences found between Gorman 0 and 1 . 
Page 20 of 23

20

${ }_{\S}$ significant differences found only between class 3 and any other class of diplopia and controls. 
Table 3. Changes of the motility parameters (in degrees) and of the Clinical Activity Score in patients responding or not responding to intravenous steroid therapy

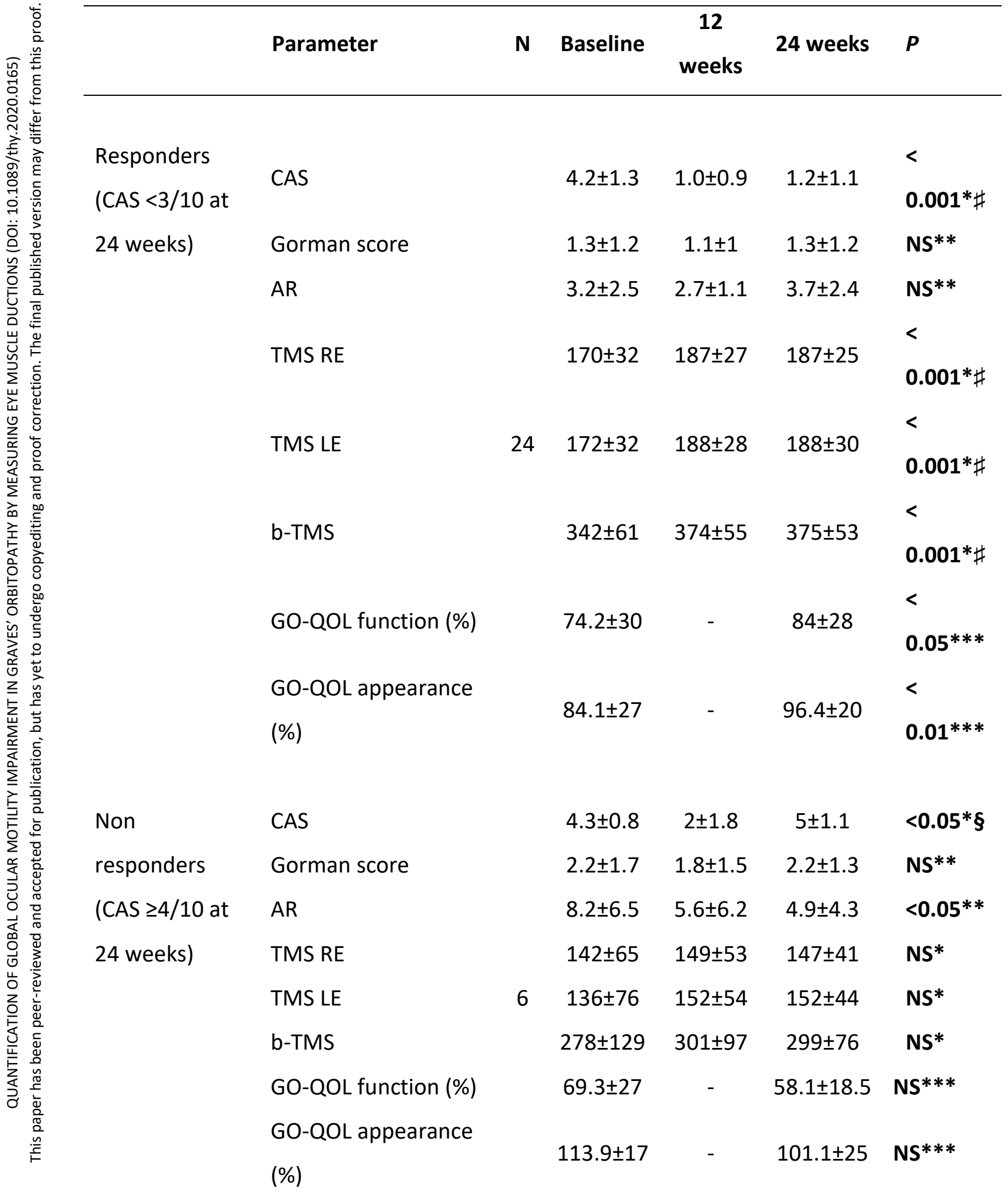


$\mathrm{RE}=$ right eye; $\mathrm{LE}=$ left eye; TMS =total motility score; $\mathrm{b}$-TMS = bi-ocular TMS; AR =asymmetry ratio, CAS = Clinical Activity Score, GO-QoL = Graves' Orbitopathy Quality Of Life questionnaire; SD = standard deviation \#: 0 vs 12 and 24 weeks; §: 12 vs 24

* Repeated Measures ANOVA, ** Friedman test, *** Wilcoxon signed rank, Values are presented as mean \pm standard deviation, unless otherwise noted. 
Table 4. Clinical outcomes of squint surgery: Gorman diplopia score, TMS and b-TMS (in degrees), AR and GO-Qol at baseline and 3 months after strabismus surgery in 66 GO patients affected with constant diplopia (Group 4).

\begin{tabular}{|c|c|c|c|c|c|}
\hline & Parameter & $\mathbf{N}$ & Baseline & 3 months & $P$ \\
\hline \multirow{6}{*}{$\begin{array}{l}\text { Patients } \\
\text { responding to } \\
\text { one surgery }\end{array}$} & Gorman score & \multirow{6}{*}{36} & $3 \pm 0$ & $0.94 \pm 0.81$ & $<0.001$ \\
\hline & Mean* TMS & & $176.5 \pm 28.9$ & $182.3 \pm 26.2$ & $<0.005$ \\
\hline & b-TMS & & $359.4 \pm 47.7$ & $371.1 \pm 46.2$ & $<0.005$ \\
\hline & AR & & $5.7 \pm 3.5$ & $3.8 \pm 3.2$ & $<0.001$ \\
\hline & GO-QOL function (\%) & & $47.4 \pm 27.3$ & $76.6 \pm 17.6$ & $<0.01$ \\
\hline & GO-QOL appearance (\%) & & $61.5 \pm 24.8$ & $81.9 \pm 19.4$ & $<0.05$ \\
\hline \multirow{6}{*}{$\begin{array}{l}\text { Patients with } \\
\text { persistent } \\
\text { diplopia after } \\
\text { one surgery }\end{array}$} & Gorman score & \multirow{6}{*}{30} & $3 \pm 0$ & $3 \pm 0$ & NS \\
\hline & Mean* TMS & & $159.6 \pm 25.3$ & $171.2 \pm 23.2$ & $<0.001$ \\
\hline & b-TMS & & $332.4 \pm 43$ & $346 \pm 43.2$ & $<0.001$ \\
\hline & $A R$ & & $5.1 \pm 4.7$ & $4.2 \pm 3.2$ & NS \\
\hline & GO-QOL function (\%) & & $52.1 \pm 24.8$ & $71.3 \pm 20.6$ & NS \\
\hline & GO-QOL appearance (\%) & & $63.9 \pm 24.2$ & $76.3 \pm 35.7$ & NS \\
\hline
\end{tabular}

TMS = total motility score, $\mathrm{b}-\mathrm{TMS}=$ biocular TMS; AR = asymmetry ratio, CAS = Clinical Activity Score, SD = standard deviation, GO-QoL = Graves' orbitopathy quality of life questionnaire.

Test: Wilcoxon signed rank

* mean of the eyes submitted to surgery; Values are shown as mean \pm SD, unless otherwise noted. 